\title{
Evaluating Characteristic Parameters for Carbon Dioxide in the Sanchez-Lacombe Equation of State
}

\author{
Kier von Konigslow, ${ }^{\dagger}$ Chul B. Park, ${ }^{\ddagger}$ and Russell B. Thompson* ${ }^{\dagger} \dagger$
}

${ }^{\dagger}$ Department of Physics and Astronomy and Waterloo Institute for Nanotechnology, University of Waterloo, 200 University Avenue West, Waterloo, Ontario, Canada N2L 3G1

${ }^{\ddagger}$ Department of Mechanical and Industrial Engineering, University of Toronto, 5 King's College Road, Toronto, Ontario, Canada M5S 3G8

ABSTRACT: For many different pure substances, large numbers of competing characteristic parameter sets exist in the literature for the Sanchez-Lacombe equation of state. This is due in part to differing research requirements or differing procedures used for determining the parameters. The existing parameters for carbon dioxide are reviewed in order to determine whether a single set of parameters can describe the equation of state over large ranges of temperature and pressure. It is found that by consideration of a large collection of experiments, a good fit can be achieved over much larger temperature and pressure ranges than previously thought possible. Properties directly related to the equation of state, such as the thermal expansivity and isothermal compressibility, are also predicted well; however, as expected, properties that depend on the internal degrees of freedom of molecules, such as the specific heats, do not correlate well. Closely agreeing parameter sets are found in the literature that fit

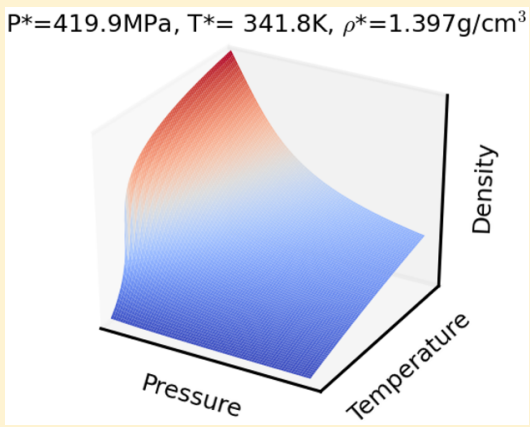
the equation of state data reasonably well over a large range. A new set of parameters is found using a least-squares approach over the largest ranges of temperature and pressure to date. These parameters are found to be $P^{*}=419.9 \mathrm{MPa}, \mathrm{T}^{*}=341.8 \mathrm{~K}$, and $\rho^{*}=1.397 \mathrm{~g} / \mathrm{cm}^{3}$ using $N=556$ experimental data points over the temperature range of $216.58-1800 \mathrm{~K}$ and the pressure range of $0.5-66.57 \mathrm{MPa}$.

\section{INTRODUCTION}

Knowledge of the pressure-volume-temperature (PVT) properties of carbon dioxide is important to many industrial and scientific applications. In industry, $\mathrm{CO}_{2}$ is frequently used as a solvent. In particular, its relatively low critical point contributes to its use in supercritical fluid extraction. ${ }^{1,2}$ In scientific applications, its abundance and well-studied properties make it desirable as a test fluid. ${ }^{1}$ With environmental concerns gaining in importance, replacing polymer foam blowing agents such as chlorofluorocarbons and hydrofluorocarbons with more environmentally friendly gases such as carbon dioxide and nitrogen becomes ever more important. $^{3-8}$ Increasingly, these applications require prediction of properties under high-pressure processing conditions. $^{9-18}$

Since its introduction in 1974, the Sanchez-Lacombe equation of state (SL-EOS) has frequently been employed to predict the $P V T$ properties of pure fluids and fluid mixtures, ${ }^{19-21}$ albeit with impaired accuracy at increased pressures for the latter. ${ }^{10,14}$ For example, in polymeric foams, the SL-EOS for mixtures has been frequently fitted to the solubility and interfacial tension of $\mathrm{CO}_{2}$ in matrix polymers. ${ }^{11,13,15-17,22}$ The SL-EOS is a popular choice for the description of the PVT properties of polymers in particular because the model includes the size of the molecules very simply through the translational entropy. In fact, the SL-EOS is one of the simplest equations of state that has a microscopic, statistical mechanical basis. Recently, the ability to independently determine the solubility and swelling of polymer/blowing agent mixtures from direct experiments has revealed serious shortcomings in the mixture SL-EOS that will need attention going forward. ${ }^{14,23-25}$ Part of this mismatch between SL theory and experiment may come from inappropriate pure-component SL-EOS parameters. Bashir et al. ${ }^{26}$ have commented on the need for accurate pure-component parameters as a prerequisite for good mixture results. Other examples of the application of the SL-EOS to $\mathrm{CO}_{2}$ systems range from polymer blends ${ }^{18}$ to pharmaceutical microencapsulation for drug delivery. ${ }^{27}$ The SLEOS has also been implicitly used for surface tension and cell density calculations of polymer foams in an inhomogeneous context through self-consistent field theory (SCFT). ${ }^{28-32}$ The SL-EOS is the homogeneous limit of the Hong and Noolandi $(\mathrm{HN})$ version of SCFT, ${ }^{33,34}$ and therefore, testing the quantitative validity of the SL-EOS for $\mathrm{CO}_{2}$ or other blowing agents is a necessary prerequisite for the extension of more complicated theories such as HN-SCFT to the structural details of polymeric foams.

The SL-EOS requires the input of experimental data to extract material-specific molecular information. ${ }^{20,35}$ Extracting that information can be done by fitting using a variety of

Received: August 23, 2016

Accepted: December 23, 2016

Published: January 9, 2017 
possible methods depending on the nature of the available experimental data as well as the properties of the material being studied. ${ }^{20,36}$ The great variety in fitting procedures has led to a large collection of characteristic parameters.,36-43 Conventional wisdom has been that a set of characteristic parameters will lead to poor predictions when used beyond the thermodynamic data range that gave rise to it. ${ }^{3,20,36,37,40}$ The implication is that because of the temperature and pressure dependence of the characteristic parameters not included in the model, the set of parameters must be found only for the range of pressures and temperatures needed for a specific application. However, for predictive applications in mixtures involving an exploration of conditions outside one small range, the use of multiple parameter sets for $\mathrm{CO}_{2}$ is impractical (large numbers of fitting parameters) and arbitrary (no protocol for deciding how many different parameter sets for $\mathrm{CO}_{2}$ to use). This is especially important as newer, more exotic materials are explored and industrialized, such as nanocellular foams, where extreme conditions of pressure and temperature are used to create these nanotechnological materials using $\mathrm{CO}_{2}$ as an environmentally benign blowing agent. In spite of this, no study has been performed that compares the existing sets of parameters to a large collection of experimental data to evaluate the predictive performance of the SL-EOS for $\mathrm{CO}_{2}$.

In this work, a review of the available sets of SL characteristic parameters for carbon dioxide was performed over a large set of experimental PVT data. From the results, it is clear that the sets of parameters found using larger ranges of temperature and pressure produce the best fits according to a standard leastsquares measure. A new parameter set for carbon dioxide, based on the largest experimental ranges of temperature and pressure to date, is found to be $P^{*}=419.9 \mathrm{MPa}, T^{*}=341.8 \mathrm{~K}$, and $\rho^{*}=$ $1.397 \mathrm{~g} / \mathrm{cm}^{3}$.

\section{THEORY}

The Sanchez-Lacombe theory is a lattice fluid model that expresses the free energy of a system by treating the fluid as a collection of lattice elements, known as mers. ${ }^{19-21,44}$ In the pure fluid, each lattice site occupies a volume $v^{*}$, with $r$ bonded mers making up a molecule of volume $r v^{*}$. The model allows for unoccupied sites, known as holes, which represent the free volume. The size of the holes, and thus the contribution to the entropy, is found through fitting to experimental data. Holes also allow for the treatment of pressure and density in the lattice construction. Interactions are restricted to nearest neighbors, with the only nonzero interaction being between mer-mer pairs.

Using the mean-field approximation to make it tractable, the above model yields a reduced free energy, scaled to be intensive and dimensionless for convenience, where the scaled functions of state are given by

$$
\begin{aligned}
& \tilde{P}=P / P^{*} \\
& \tilde{T}=T / T^{*} \\
& \tilde{\rho}=\rho / \rho^{*}=V^{*} / V
\end{aligned}
$$

The scaled parameters are defined in terms of the characteristic parameters

$$
\begin{aligned}
& T^{*}=\epsilon^{*} / k_{\mathrm{B}} \\
& P^{*}=\epsilon^{*} / v^{*}
\end{aligned}
$$

$$
\rho^{*}=M / r v^{*}
$$

where $\epsilon^{*}$ characterizes the mer-mer interaction (with dimensions of energy), $k_{\mathrm{B}}$ is Boltzmann's constant, and $M$ is the molecular weight. The number of mer segments per molecule is related to the characteristic parameters by ${ }^{20}$

$$
k_{\mathrm{B}} T^{*} \rho^{*} / P^{*}=v^{*} \rho^{*}=M / r
$$

Thus, the pure-component fluid can be characterized by a set of three characteristic parameters along with the molecular weight. In the literature, these characteristic parameters have been predominantly the set $\epsilon^{*}, v^{*}$, and $r$ or, alternatively, $P^{*}, T^{*}$, and $\rho^{*}$. In practice, many such sets of parameters can be used provided they span the parameter space.

The free energy yields the SL-EOS for pure fluids, given by

$$
\tilde{P}+\tilde{\rho}^{2}+\tilde{T}\left[\ln (1-\tilde{\rho})+\left(1-\frac{1}{r}\right) \tilde{\rho}\right]=0
$$

A full derivation of the equation of state from the model can be found in ref 20 .

The characteristic parameters are found by fitting the equation of state to experimentally obtained thermodynamic data. Sanchez and Lacombe proposed several possibilities for such fitting in order to take advantage of existing experimental data. ${ }^{20}$ For nonpolymeric materials that experience a region of two-phase coexistence, Sanchez and Lacombe proposed a method of determining the parameters using saturated vapor pressure data that minimized computational work while taking advantage of the large body of existing data. ${ }^{20}$ These computational savings are based on a reduction of the threeparameter nonlinear least-squares fit to a single-parameter fit using a small number of experimentally determined data points (i.e., the normal boiling point and the midpoint of the liquidvapor coexistence curve). ${ }^{20}$ This approach should be used with caution, as the simplified fitting procedure may sacrifice the exactness of the fit while modern computing has made the numerical savings moot. For polymeric materials as well as supercritical fluids, inferring parameters from saturated vapor pressure is not possible. For carbon dioxide, Pope et al. ${ }^{42}$ simplified the fit of the characteristic parameters by eliminating the least-squares method entirely, using instead a calculation based on the experimentally determined vapor and liquid densities at the normal boiling point and the critical temperature. Hariharan et al. ${ }^{36}$ questioned the characteristic parameters found by Pope et al. since carbon dioxide does not have a boiling point at atmospheric pressure. The approach of Pope et al. should also be used with caution because mean-field theories, such as the SL-EOS, are not able to correctly predict behavior near the critical point. ${ }^{20,45,46}$ Heidemann and coworkers ${ }^{47,48}$ suggested modifications to the SL-EOS to allow it to correctly predict both the critical point and data away from the critical point. Reversing this, it allowed them to use the critical temperature together with the critical pressure and acentric factor to extract the characteristic parameters. They added a Péneloux volume translation term ${ }^{49}$ to allow for a good fit both at the critical point and away from it, and in some cases they added a temperature dependence to one of the characteristic parameters. This modified EOS is distinct from the SL-EOS, although related to it, and one therefore anticipates different characteristic parameters. The main cost of the modifications and the parameter temperature dependence is that one has to abandon the connection of the parameters with the underlying statistical mechanical model. 
Table 1. List of Pure-Component $\mathrm{CO}_{2}$ SL Parameter Sets with Corresponding Pressure and Temperature Ranges, The Experimental Methods Used (Where Available), and the Reference Numbers for the Sources of the Experimental Data; The Deviations between Theory and Experiment $\left(S_{S} Q_{p}\right.$, eq 10) Are Also Given ${ }^{a}$

\begin{tabular}{|c|c|c|c|c|c|c|c|c|c|}
\hline group & year & $P^{*}(\mathrm{MPa})$ & $T^{*}(\mathrm{~K})$ & $\rho^{*}\left(\mathrm{~g} / \mathrm{cm}^{3}\right)$ & $P(\mathrm{MPa})$ & $T(\mathrm{~K})$ & method & data source ref & $\operatorname{SSQ}_{p}\left(10^{-4}\right)$ \\
\hline Kilpatrick ${ }^{39}$ & 1986 & 719.51 & 280.0 & 1.618 & $0.51-7.4$ & $216.6-304$ & - & 63 & 241.1 \\
\hline Kiszka $^{40}$ & 1988 & 574.5 & 305.0 & 1.510 & $10.1-16.2$ & $313-333$ & piezometer & 64 & 58.64 \\
\hline Pope $^{42}$ & 1991 & 659.63 & 283.0 & 1.62 & 0.1 & 304 & - & 65 & 245.1 \\
\hline Wang $^{43}$ & 1991 & 720.3 & $b$ & 1.580 & $<8$ & $313-333$ & piezometer & 64 & 424.7 \\
\hline Hariharan ${ }^{36}$ & 1993 & 418.07 & 316.0 & 1.369 & 0.58 & 219.26 & piezometer & 66 & 213.5 \\
\hline $\operatorname{Garg}^{38}$ & 1994 & 464.2 & 328.1 & 1.426 & $<26$ & $323-373$ & piezometer & 67 & 14.57 \\
\hline Xiong $^{3}$ & 1995 & 420.0 & 340.9 & 1.392 & $20-60$ & $360-420$ & viscometer & 3 & 6.083 \\
\hline Doghieri $^{56}$ & 1996 & 630.0 & 300.0 & 1.515 & $8-50$ & $270-360$ & - & 68 & 68.12 \\
\hline Nalawade ${ }^{41}$ & 2006 & 427.7 & 338.7 & 1.4055 & $<30$ & $333-420$ & buoyancy & 1 & 6.101 \\
\hline Funami $^{57}$ & 2007 & 369.1 & 341.2 & 1.2530 & $<40$ & $394.4-522.9$ & buoyancy & 57 & 95.87 \\
\hline $\mathrm{Cao}^{37}$ & 2010 & 453.53 & 327.0 & 1.46 & $13-28$ & $318-368$ & - & 69 & 18.78 \\
\hline $\operatorname{Arce}^{58}$ & 2009 & 585.61 & 301.23 & 1.53253 & $<7.4$ & $216.6-304.0$ & - & 58 & 76.94 \\
\hline this work & 2016 & 419.9 & 341.8 & 1.397 & $<66.57$ & $216.6-1100.0$ & - & - & 4.968 \\
\hline
\end{tabular}

${ }^{a}$ All of the experimental data were derived from pure-component samples. ${ }^{b}$ The temperature-scaling parameter of Wang et al. is given by the expression $T^{*}=208.9+0.459 T-7.56 \times 10^{-4} T^{2}$.
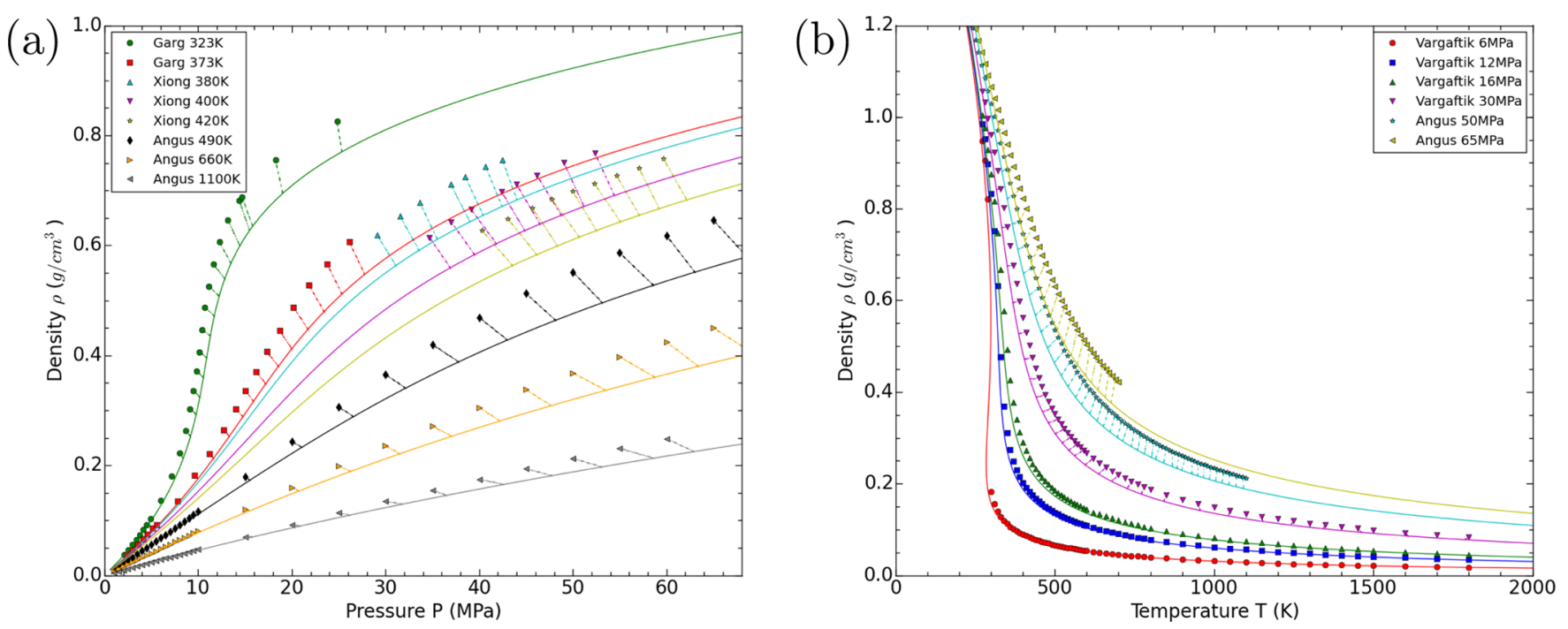

Figure 1. Comparisons of (a) density-pressure isotherms and (b) density-temperature isobars calculated using the Kilpatrick and Chang ${ }^{39}$ parameters with those of experiment. Solid lines represent theory and filled circles represent experiment at (green @) $323 \mathrm{~K}$, (red $\mathbf{\square}) 373 \mathrm{~K}$, (cyan A) $380 \mathrm{~K}$, (magenta $\boldsymbol{\nabla}) 400 \mathrm{~K}$, (yellow $\star$ ) $420 \mathrm{~K}$, (black $>$ ) $490 \mathrm{~K}$, (orange $>$ ) $660 \mathrm{~K}$, and (gray $1100 \mathrm{~K}$. Dashed lines have been added to link the experimental data with the corresponding theoretical curves for clarity. The legends indicate the sources of the experimental data.

This can lead to other problems in the context of mixtures, where binary fitting parameters must start to take on temperature dependences that do not arise from the original statistical mechanical models in order to match experimental data. The modified SL-EOS of Heidemann and co-workers has the advantage of speed and accuracy in certain contexts, which makes it excellent for some industrial applications. Machida and co-workers have also proposed a temperature-dependence modification of the SL-EOS and applied it to systems including $\mathrm{CO}_{2}{ }^{50,51}$ Since these modified SL equations represent essentially different theories, they are not expected to result in the same characteristic parameters as the standard SL-EOS.

The usual method for determining characteristic parameters is to use a nonlinear least-squares approach. ${ }^{3,36-41}$ Typically, the fitting is done by minimizing a measure similar to

$$
\begin{aligned}
\operatorname{SSQ}_{\rho}= & \sum_{i=1}^{N_{\mathrm{sp}}}\left[\frac{\rho_{\mathrm{t}}\left(T_{i}, P_{i}\right)-\rho_{\mathrm{e}, i}}{\rho_{\mathrm{e}, i}}\right]^{2}+\sum_{j=1}^{N_{\mathrm{coex}}}\left\{\left[\frac{\rho_{\mathrm{t}}^{\mathrm{l}}\left(T_{\mathrm{j}}\right)-\rho_{\mathrm{e}, j}^{1}}{\rho_{\mathrm{e}, j}^{\mathrm{l}}}\right]^{2}\right. \\
& \left.+\left[\frac{\rho_{\mathrm{t}}^{\mathrm{g}}\left(T_{\mathrm{j}}\right)-\rho_{\mathrm{e}, j}^{\mathrm{g}}}{\rho_{\mathrm{e}, j}^{\mathrm{g}}}\right]^{2}\right\}
\end{aligned}
$$

where $\rho_{\mathrm{e}, i}$ is the $i$ th experimentally determined density measured at temperature $T_{i}$ and pressure $P_{i}, \rho_{\mathrm{t}}$ is the theoretically predicted density at the same pressure and temperature, $N_{\mathrm{sp}}=364$ is the total number of experimental data points in both the single-phase and supercritical regions, $\rho_{\mathrm{e}, j}^{1}$ and $\rho_{\mathrm{e}, j}^{\mathrm{g}}$ are the $j$ th experimentally determined densities of the liquid and gas, respectively, along the line of liquid-vapor coexistence, $\rho_{\mathrm{t}}^{1}$ and $\rho_{\mathrm{t}}^{\mathrm{g}}$ are the theoretically predicted densities of the coexisting liquid and vapor, respectively, at temperature $T_{j}$, and $N_{\text {coex }}=200$ is the number of data points on the coexistence 

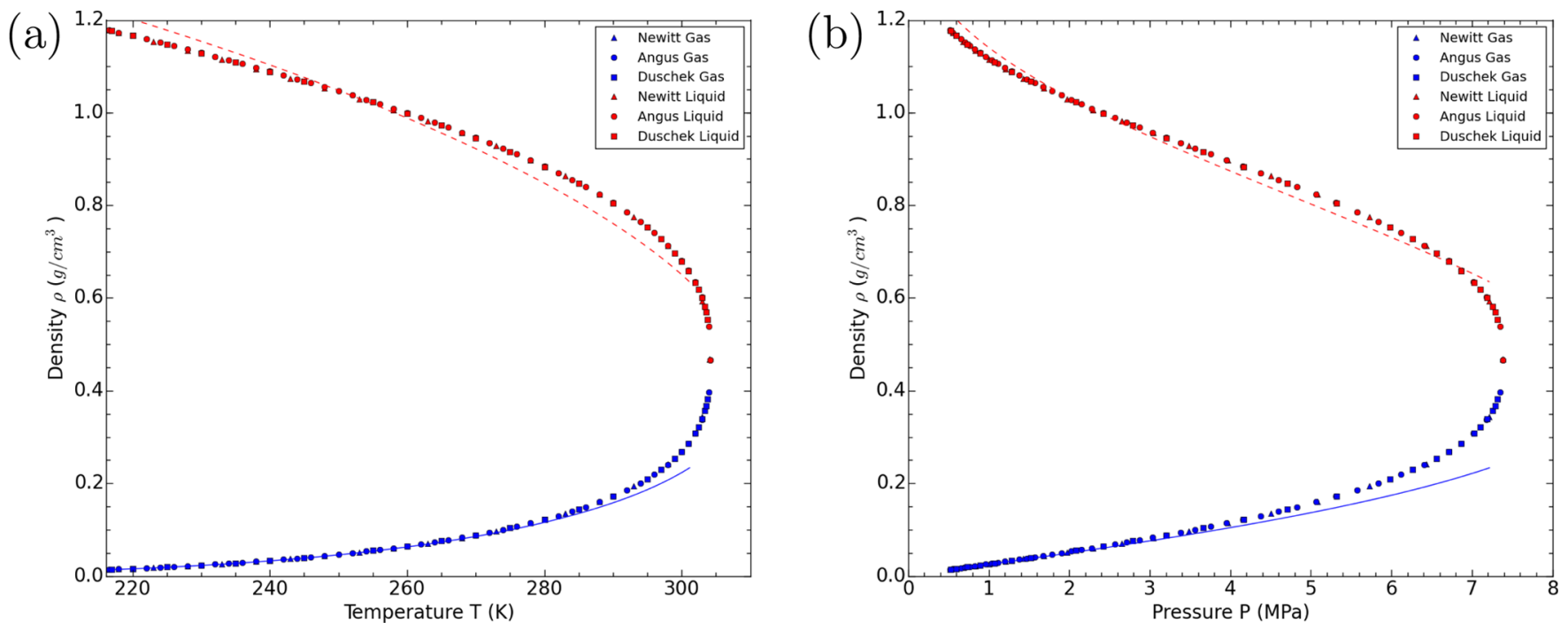

Figure 2. Comparisons of (a) the saturated liquid-vapor density-temperature curve and (b) the density-pressure curve obtained using the Kilpatrick and Chang ${ }^{39}$ parameters with the ones obtained experimentally. Lines represent predicted vapor (blue solid) and liquid (red dashed) densities, while filled shapes represent experiment for both vapor (blue) and liquid (red). The legends indicate the sources of experimental data.

curve. The minimization can be performed in many ways, including the Levenberg-Marquardt algorithm. ${ }^{52,53}$ The sum of the squares of the fractional differences (SSQ) between the experimental data and theoretical prediction is used to measure the goodness of fit. The sum is calculated over the range of available pressures and temperatures.

Although there are some similarities among the fitting methods of many groups, it has been noted in the past that variations in fitting practices can significantly affect the results. In order to obtain a set of SL characteristic parameters for polypropylene, Zoller ${ }^{54}$ first performed a fit to the zeropressure isobar to determine $T^{*}$ and $\rho^{*}$ and then obtained $P^{*}$ from an average of values calculated by comparing theoretical and experimental isothermal compressibility values at zero pressure over multiple temperatures. Pottiger and Laurence ${ }^{55}$ found that the fitting method employed by Zoller emphasized the fit at lower pressures at the expense of the fit at high pressures. They concluded that the poor predictive power of the theory was attributable to the fitting procedure and found that a good fit was possible by simultaneously solving for all three parameters using a nonlinear least-squares method.

\section{DISCUSSION}

There exists in the literature a belief that no constant set of SLEOS parameters can be used to predict thermodynamic data over wide ranges of pressure and temperature. Despite this expectation, no universally accepted limits exist on the pressure and temperature ranges appropriately represented by a single set of SL-EOS parameters. Because of this, many groups have proposed different parameters for carbon dioxide with different scopes and overlapping ranges. ${ }^{3,36-43,56-58}$ Great care, however, must be taken when applying a nonlinear leastsquares fitting algorithm. Fitting algorithms based on leastsquares methods are famously sensitive to outliers, which can be amplified by decreased numbers of data points. ${ }^{59}$ They are also prone to finding local rather than global minima with respect to parameters. ${ }^{60-62}$ Furthermore, care must be taken when extrapolating fitted nonlinear functions, since curve features outside of the fitted range may be poorly predicted. These pitfalls can be mitigated by increasing the scope and

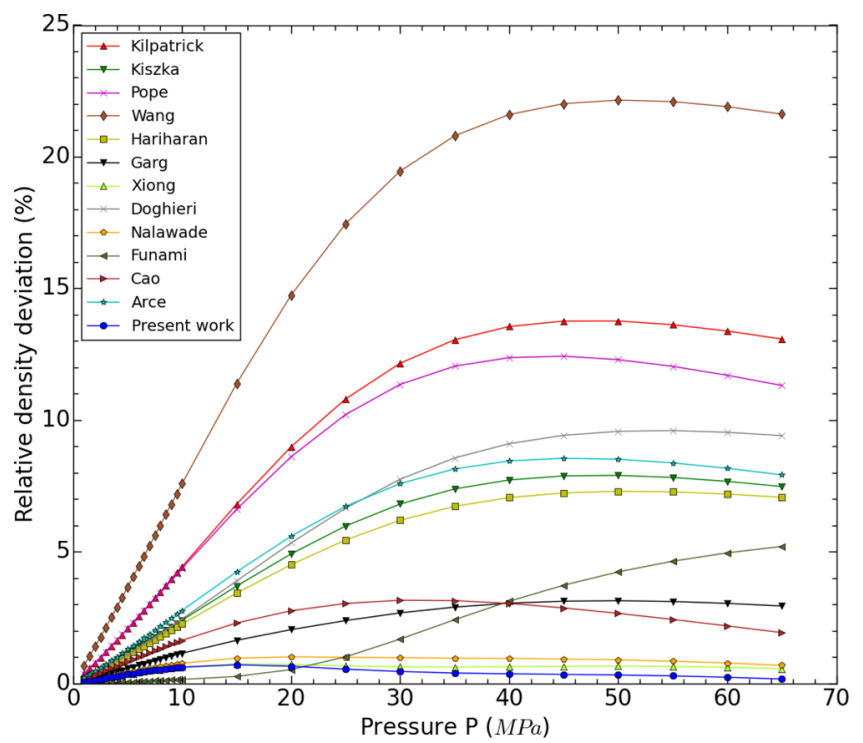

Figure 3. Comparison of relative density deviations between experiment and theory as functions of pressure at a temperature of $490 \mathrm{~K}$ for all sets of characteristic parameters given in Table 1 . The legend indicates the source for each parameter set.

Table 2. Sanchez-Lacombe Pure-Fluid Parameters for Carbon Dioxide That Fit the Experimentally Determined Thermodynamic Data ${ }^{a}$

\begin{tabular}{lcccc}
\multicolumn{1}{c}{ group } & $P^{*}(\mathrm{MPa})$ & $\mathrm{T}^{*}(\mathrm{~K})$ & $\rho^{*}\left(\mathrm{~g} / \mathrm{cm}^{3}\right)$ & $\mathrm{SSQ}_{p}\left(10^{-4}\right)$ \\
Xiong $^{3}$ & 420.0 & 340.9 & 1.392 & 6.083 \\
Nalawade $^{41}$ & 427.7 & 338.7 & 1.4055 & 6.101 \\
this work & $\mathbf{4 1 9 . 9}$ & 341.8 & $\mathbf{1 . 3 9 7}$ & $\mathbf{4 . 9 6 8}$
\end{tabular}

${ }^{a}$ Parameter choices that fit the entire range of data reasonably well fall roughly within $\pm 8 \mathrm{MPa}, \pm 3 \mathrm{~K}$, and $\pm 0.01 \mathrm{~g} / \mathrm{cm}^{3}$ of each other, but because of the nonlinear nature of the equation of state, not all parameter combinations that fall within these bounds will fit well.

number of data points used for fitting. The consequences of this can reach beyond the prediction of pure fluid properties. It has been observed in the past that incorrect polymeric pure 
(a)

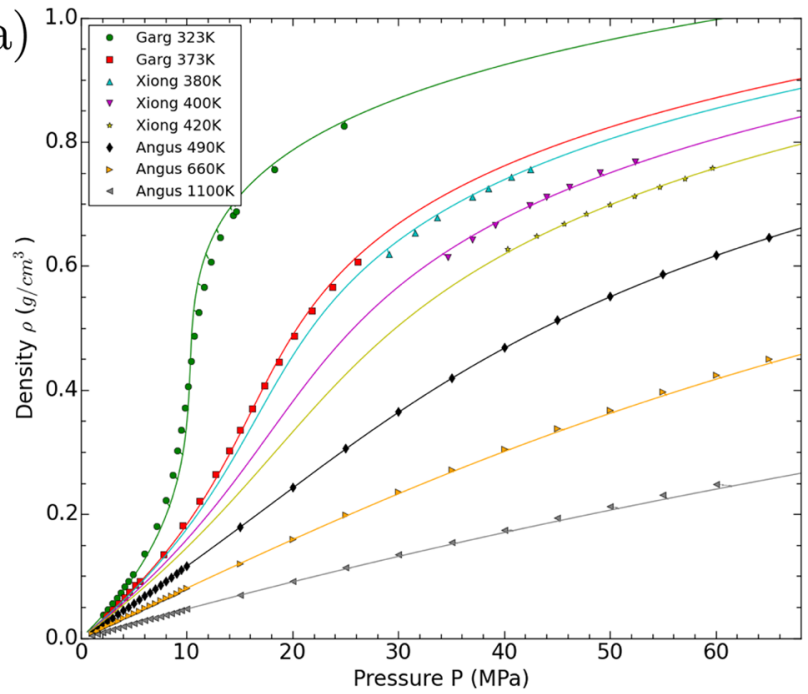

(b)

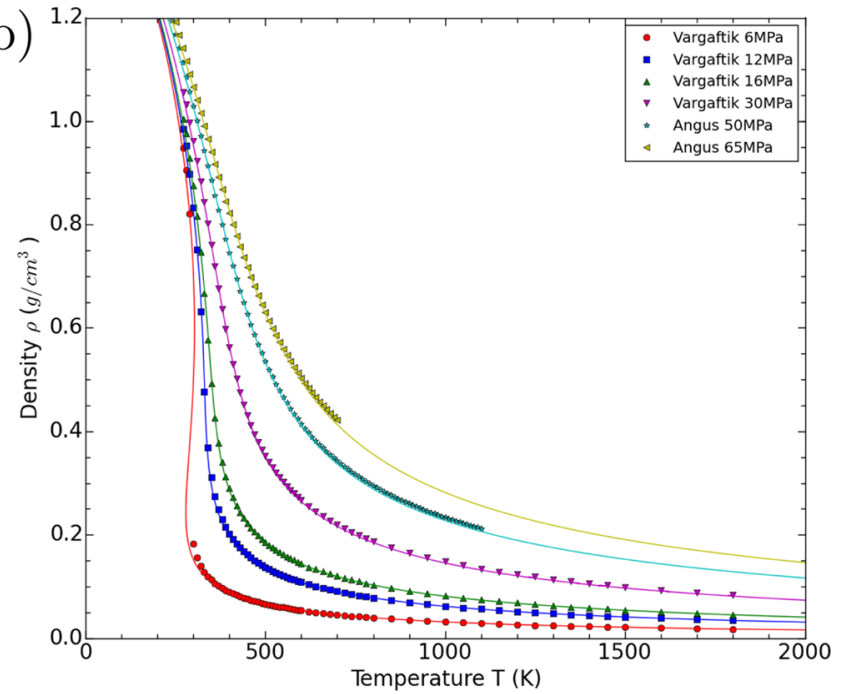

Figure 4. Comparisons of (a) density-pressure isotherms and (b) density-temperature isobars obtained using the parameters calculated in this work with those obtained experimentally. Solid lines represent theory and filled circles represent experiment at (green 0) $323 \mathrm{~K}$, (red $\mathbf{\square}$ ) $373 \mathrm{~K}$, $($ cyan $\mathbf{\Delta}) 380 \mathrm{~K}$, (magenta $\boldsymbol{\nabla}$ ) $400 \mathrm{~K}$, (yellow $\star$ ) $420 \mathrm{~K}$, (black $\bullet$ ) $490 \mathrm{~K}$, (orange $>$ ) $660 \mathrm{~K}$, and (gray $>$ ) $1100 \mathrm{~K}$. The legends indicate the sources of experimental data.
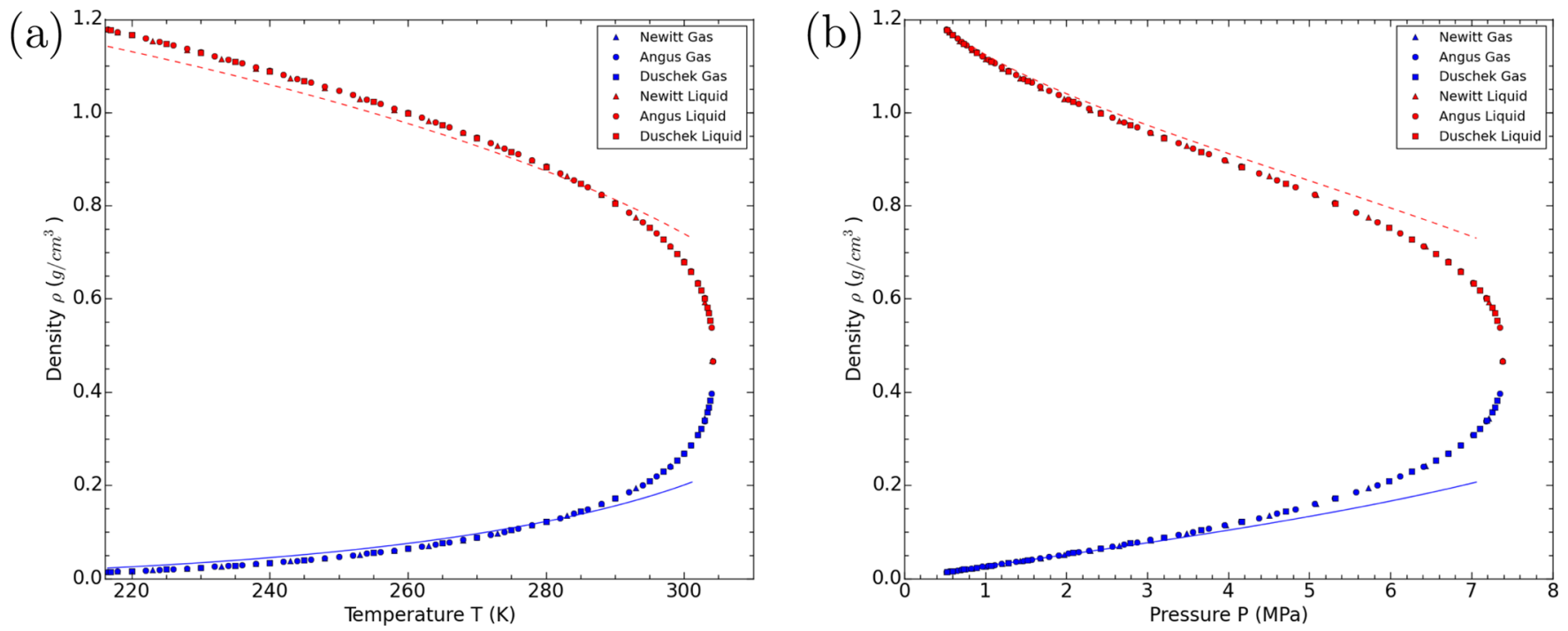

Figure 5. Comparisons of (a) the saturated liquid-vapor density-temperature curve and (b) the density-pressure curve obtained using the parameters calculated in this work with the ones obtained experimentally. Lines represent predicted vapor (blue solid) and liquid (red dashed) densities, while filled shapes represent experiment for both vapor (blue) and liquid (red). The legends indicate the sources of experimental data.

fluid parameters lead to unsatisfactory predictions of the behavior of polymeric mixtures. ${ }^{26,36,55}$ Table 1 lists some of the competing SL-EOS parameter sets for carbon dioxide as well as the experimental source, method, and temperature and pressure ranges of the data from which the parameters are regressed. It should be noted that the nonlinearity of the SL-EOS means that small differences in individual parameters can have a significant effect on the predictions of the theory.

It is also important to consider the quality of different data sets when applying a least-squares algorithm so that appropriate weights can be assigned to each. For the sets listed in Table 1, few error bounds were given, so there is little basis for weighting the data differently. Nonetheless, despite the use of differing experimental methods, the various results show high levels of consistency. ${ }^{64}$
The fitting procedures employed are as varied as the ranges of the experimental data. Kilpatrick and Chang ${ }^{39}$ performed a nonlinear least-squares fit to the vapor pressure and saturated densities of $\mathrm{CO}_{2}$ along the line of liquid-vapor coexistence. Hariharan et al. ${ }^{36}$ determined their parameters using a fit to the known critical temperature of $\mathrm{CO}_{2}$ as well as from the liquid density, gas density, and heat of vaporization at a single arbitrarily chosen coexistence vapor pressure and temperature. Xiong and $\mathrm{Kiran}^{3}$ do not describe their fitting procedure in detail but mention that they obtained their parameters from an optimization of PVT data over the temperature and pressure range listed in Table 1 .

Figures 1 to 5 compare the SL theoretical density-pressure isotherms, density-temperature isobars, and coexistence curves with experiment. Experimentally obtained thermodynamic data was taken from the literature. ${ }^{3,38,64,68,70,71}$ Figure 1 compares 


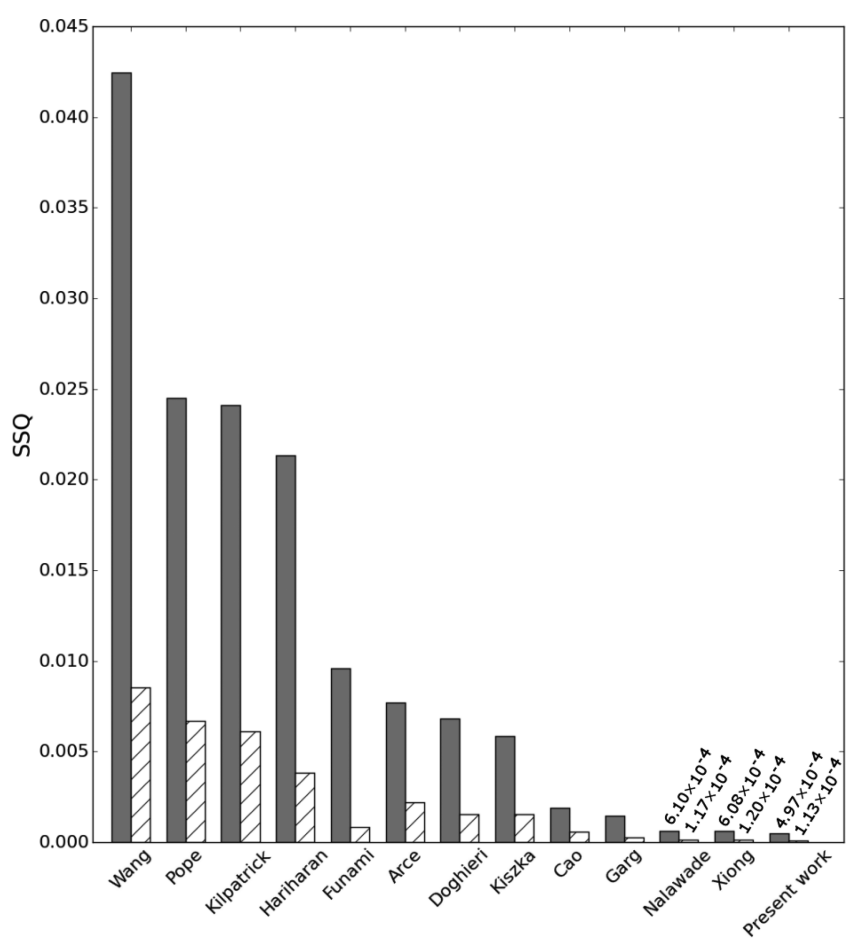

Figure 6. Comparison of the sums of the squares of the fractional deviations predicted by the different sets of SL parameters from experiment using the pressure-based measure given by eq 10 (solid bars) and the more standard density-based measure given by eq 9 (hashed bars) for all data points. The parameter sets that fit the data well are given in Table 2 .

Table 3. List of $\mathrm{CO}_{2}$ Critical Temperatures and Pressures Predicted by the SL Parameters of Various Groups; The Experimentally Determined Critical Point is $304.2 \mathrm{~K}$ at 7.38 $\mathrm{MPa}^{2,64}$

\begin{tabular}{lcc}
\multicolumn{1}{c}{ group } & $T_{\mathrm{c}}(\mathrm{K})$ & $P_{\mathrm{c}}(\mathrm{MPa})$ \\
\hline Kilpatrick $^{39}$ & 309.7 & 8.66 \\
Kiszka $^{40}$ & 316.2 & 9.08 \\
Pope $^{42}$ & 305.0 & 8.89 \\
Wang $^{43}$ & 310.4 & 8.38 \\
Hariharan $^{36}$ & 303.9 & 8.73 \\
Garg $^{38}$ & 318.1 & 9.42 \\
Xiong $^{3}$ & 319.0 & 9.64 \\
Doghieri $^{56}$ & 320.1 & 8.85 \\
Nalawade $^{41}$ & 318.5 & 9.66 \\
Funami $^{57}$ & 316.8 & 8.69 \\
Cao $^{37}$ & 312.8 & 9.65 \\
Arce $^{58}$ & 313.7 & 9.09 \\
this work $^{5}$ & 319.2 & 9.70 \\
\hline
\end{tabular}

the PVT predictions made using the Kilpatrick and Chang ${ }^{39}$ parameters with experiment, while Figure 2 compares predicted coexistence data with experiment, again using the Kilpatrick and Chang parameters. It is clear from the figures that the predictions agree with experiment in the coexistence region of 216-304 K (the source of their fitting data) but do very poorly at high pressures and high temperatures. While other parameter sets suffer the same limitation, the Kilpatrick and Chang parameters were chosen here for the purpose of illustration. Figure 3 shows the relative density deviations between theory and experiment at an example temperature of $490 \mathrm{~K}$ for all of the parameter sets.
The parameter sets of Xiong and $\mathrm{Kiran}^{3}$ and Nalawade et $\mathrm{al}^{41}$ are very similar. In both instances, the temperature and pressure ranges of the experimental data are larger than those of the others. Both parameter sets show good agreement between theoretically predicted PVT data and experiment. The agreement goes beyond the data sets from which the parameters were drawn.

By means of a least-squares fitting approach, new parameters have been found that fit all of the referenced thermodynamic data simultaneously. In order to include the greatest amount of thermodynamic data possible, density, temperature, and pressure data in the homogeneous liquid and vapor regions, the saturated liquid-vapor coexistence curves from the triple point to the critical point $(216.6 \mathrm{~K}$ at $0.518 \mathrm{MPa}$ and $304.2 \mathrm{~K}$ at $7.38 \mathrm{MPa}$, respectively $\left.{ }^{2,64}\right)$, and the supercritical region were gathered. In all, the new parameters were found using $N=556$ experimental data points over the temperature range of $216.58-1800 \mathrm{~K}$ and the pressure range of $0.5-66.57$ $\mathrm{MPa} .3,38,64,68,70,71$ Because of the inherent complication involved in calculating theoretical values of density using the SL-EOS because of the nonlinear dependence on the density, a sum of squares based on pressure is used instead. Forgoing nonlinear solvers greatly increases the speed of calculation as well as the accuracy of the results. This leads to a new measure of goodness of fit given by

$$
\mathrm{SSQ}_{P}=\sum_{i=1}^{N_{\mathrm{sp}}}\left[\frac{P_{\mathrm{t}}\left(T_{i}, \rho_{i}\right)-P_{\mathrm{e}, i}}{P_{\mathrm{e}, i}}\right]^{2}+\sum_{j=1}^{N_{\mathrm{coex}}}\left[\frac{P_{\mathrm{t}}^{1, \mathrm{~g}}\left(T_{j}\right)-P_{\mathrm{e}, j}^{\mathrm{l}, \mathrm{g}}}{P_{\mathrm{e}, j}^{\mathrm{l}, \mathrm{g}}}\right]^{2}
$$

where $P_{\mathrm{e}, i}$ is the $i$ th experimentally determined pressure corresponding to temperature $T_{i}$ and density $\rho_{i}, P_{\mathrm{t}}$ is the theoretically predicted pressure at the same temperature and density, $N_{\mathrm{sp}}=364$ is the total number of experimental data points in both the single-phase and supercritical regions, $P_{\mathrm{e}}^{\mathrm{l}, \mathrm{g}}$ is the $j$ th experimentally determined pressure of the coexisting liquid and vapor, and $N_{\text {coex }}=200$ is the number of data points on the coexistence curve. The theoretically predicted pressure at liquid-vapor coexistence, $P_{t}^{l, g}\left(T_{j}\right)$ is calculated at temperature $T_{j}$ using the method of equal liquid and vapor grand potentials. Since the SL-EOS is linear as a function of pressure, the new least-squares minimization does not require the use of numerical nonlinear solvers. Care has also been taken to exclude data close to both the experimental and theoretical critical points because of the breakdown of mean-field theory in those regions. Data within $15 \mathrm{~K}$ of either of the points have been omitted, following Sanchez and Lacombe, ${ }^{20}$ as well as those within 1.5 MPa. Table 2 shows the characteristic parameters calculated to minimize eq 10 as well as the literature parameters that fit the data well using eq 10 as a measure of goodness of fit. Figure 4 shows good agreement between PVT theory and experiment using the parameter set calculated in this work, in both the single-phase region and the supercritical region. Figure 5 shows reasonable agreement between the predicted coexistence curves and experiment. From Table 2, one can see that parameter choices that fit the entire range of data reasonably well fall roughly within $\pm 8 \mathrm{MPa}$, $\pm 3 \mathrm{~K}$, and $\pm 0.01 \mathrm{~g} / \mathrm{cm}^{3}$ of each other, but because of the nonlinear nature of the equation of state, not all parameter combinations that fall within these bounds will fit well. The characteristic parameters calculated in this work give a hole volume of $v^{*}=1.12 \times 10^{-23} \mathrm{~cm}^{3}$ or, equivalently, $v^{*}=6.76$ $\mathrm{cm}^{3} / \mathrm{mol}$. 
(a)

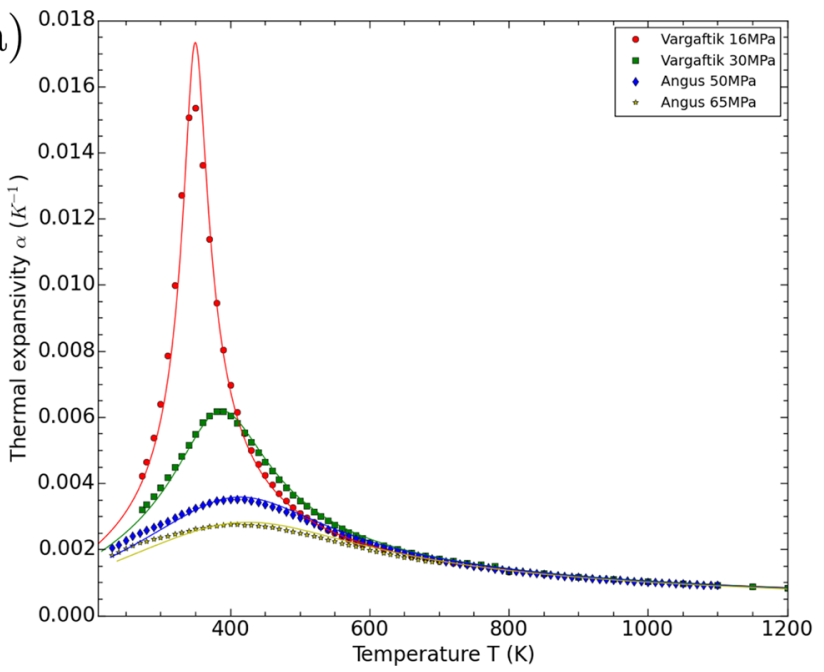

(b)

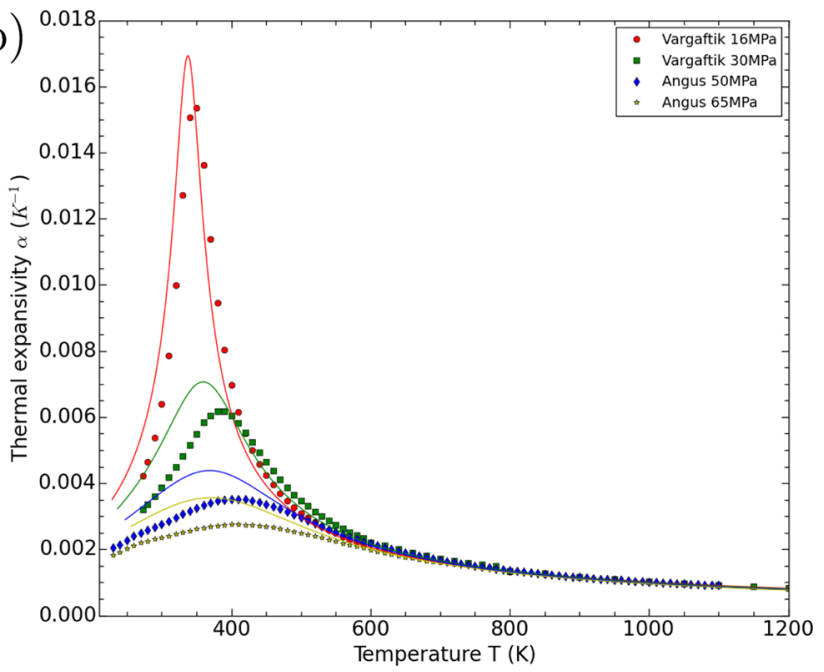

Figure 7. Thermal expansivity as a function of temperature for a selection of pressures using (a) the characteristic parameters presented in this work and (b) the characteristic parameters of Kilpatrick and Chang. ${ }^{39}$ Symbols are experimental data from the sources indicated in the legends, and the solid lines are fits using the SL expression for $\alpha$.
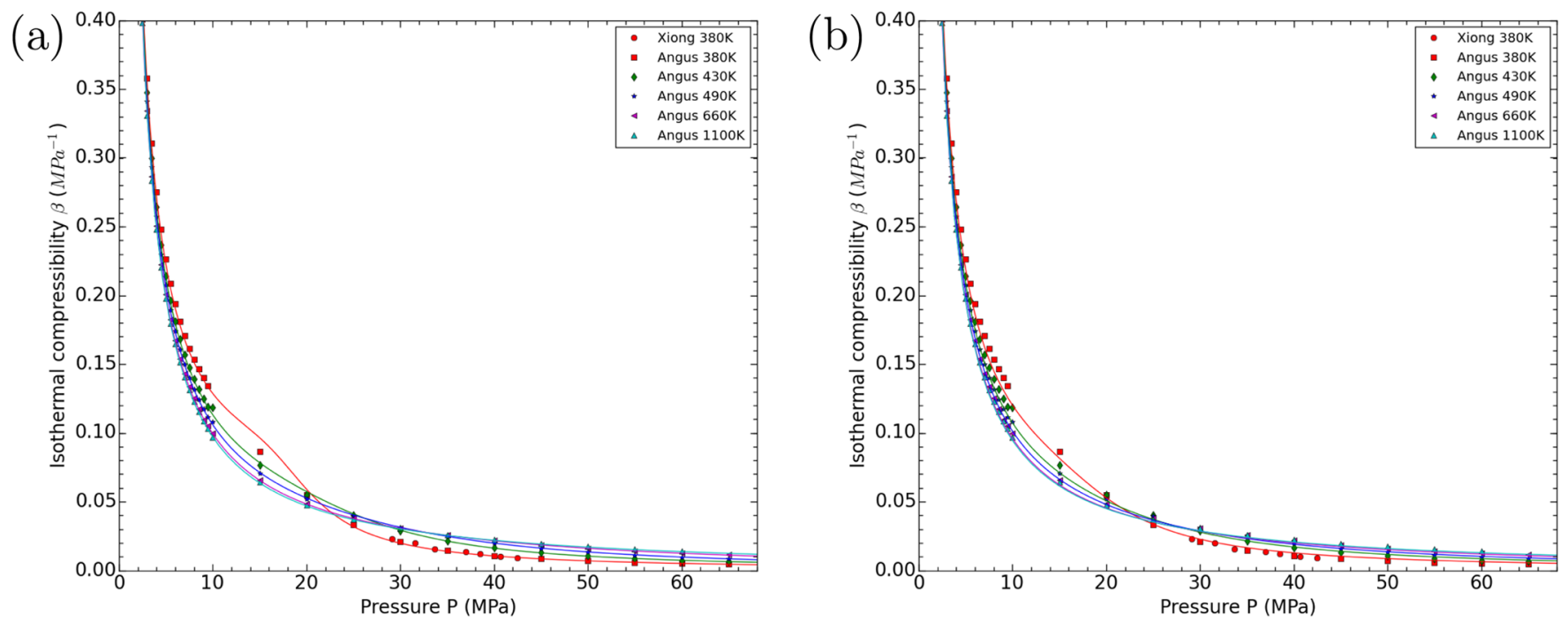

Figure 8. Isothermal compressibillity as a function of pressure for a selection of temperatures using (a) the characteristic parameters presented in this work and (b) the characteristic parameters of Kilpatrick and Chang. ${ }^{39}$ Symbols are experimental data from the sources indicated in the legends, and the solid lines are fits using the SL expression for $\beta$.

Equation 10 has been used to calculate the SSQ which is taken as a measure of the goodness of fit for each set of parameters in Table 1. The results found in Figure 6 confirm that the parameters shown in Table 2 provide the most satisfactory fits according to the least-squares measure in eq 10. Even when the more standard measure of SSQ in terms of density (eq 9) is used, Figure 6 shows that the parameters in Table 2 still produce a satisfactory fit. By either measure, the parameter set calculated in this work produces the best fit. Regardless of the least-squares measure used, one can objectively see that the parameter sets of those groups with a low SSQ as measured by eq 9 or 10 fit the data better over the entire pressure and temperature range than those with higher SSQ values. Only the parameters from this work have been evaluated using the totality of the data reviewed here.

Despite the fact that the SL equation of state allows for the calculation of the critical point, the prediction is not expected to be accurate. The SL equation of state, as a mean-field theory, breaks down near the critical point. ${ }^{20}$ A comparison of the experimentally determined critical point of carbon dioxide with those obtained using the parameter sets calculated by different groups is shown in Table 3. Not surprisingly, most of the SLcalculated critical points do not agree with the experimentally determined one, with the majority being much higher than the accepted value. The parameters calculated by Kilpatrick and Chang, ${ }^{39}$ Pope et al., ${ }^{42}$ and Hariharan et al. ${ }^{36}$ appear to be exceptions in that they do produce a relatively accurate critical temperature. In the case of Hariharan et al., this is not surprising since their fit is based in part on the critical temperature. Kilpatrick and Chang and Pope et al. also base their fits on thermodynamic data near the critical point. In all three cases, the more accurate estimation of the critical point comes at the cost of a poor fit elsewhere, as illustrated in Figure 1. Table 3 shows that they also fail to accurately predict the critical pressure. 

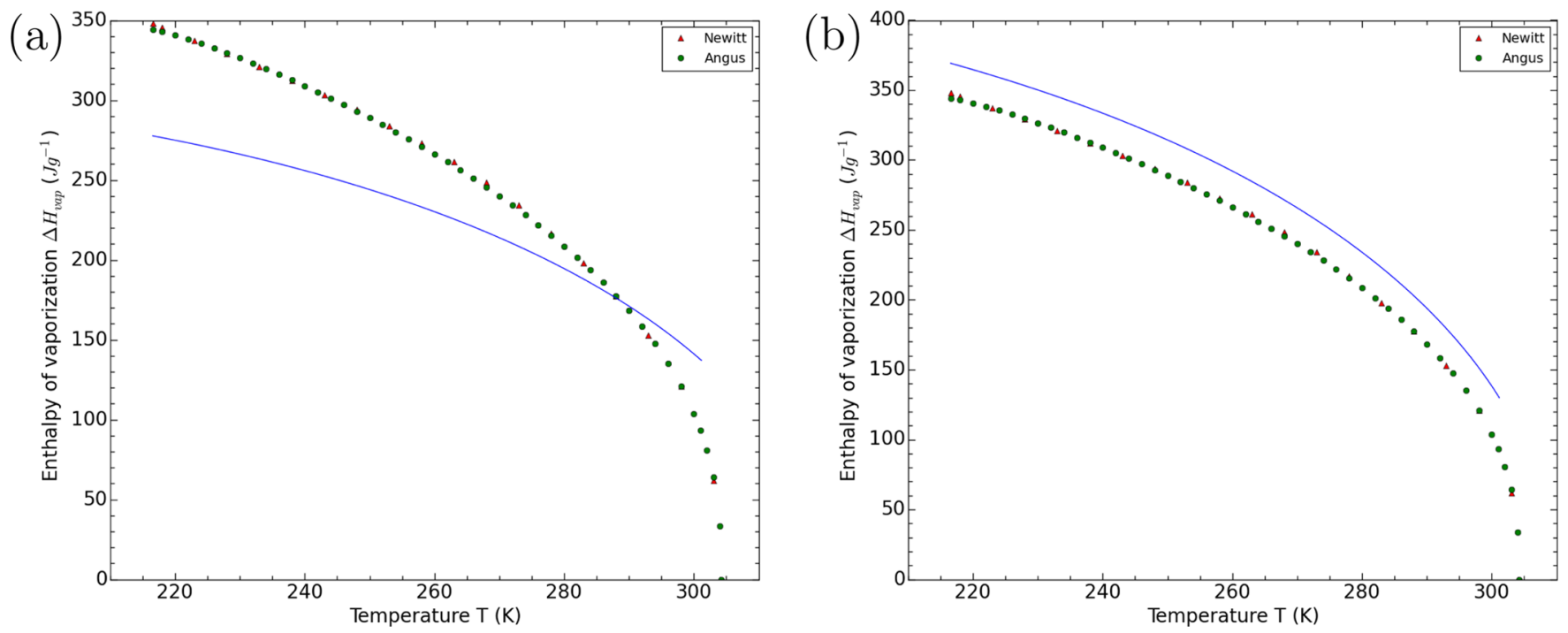

Figure 9. Enthalpy of vaporization as a function of temperature using (a) the characteristic parameters presented in this work and (b) the characteristic parameters of Kilpatrick and Chang. ${ }^{39}$ Symbols are experimental data from the sources indicated in the legends, and the solid lines are fits using the SL expression for $\Delta H_{\text {vap }}$
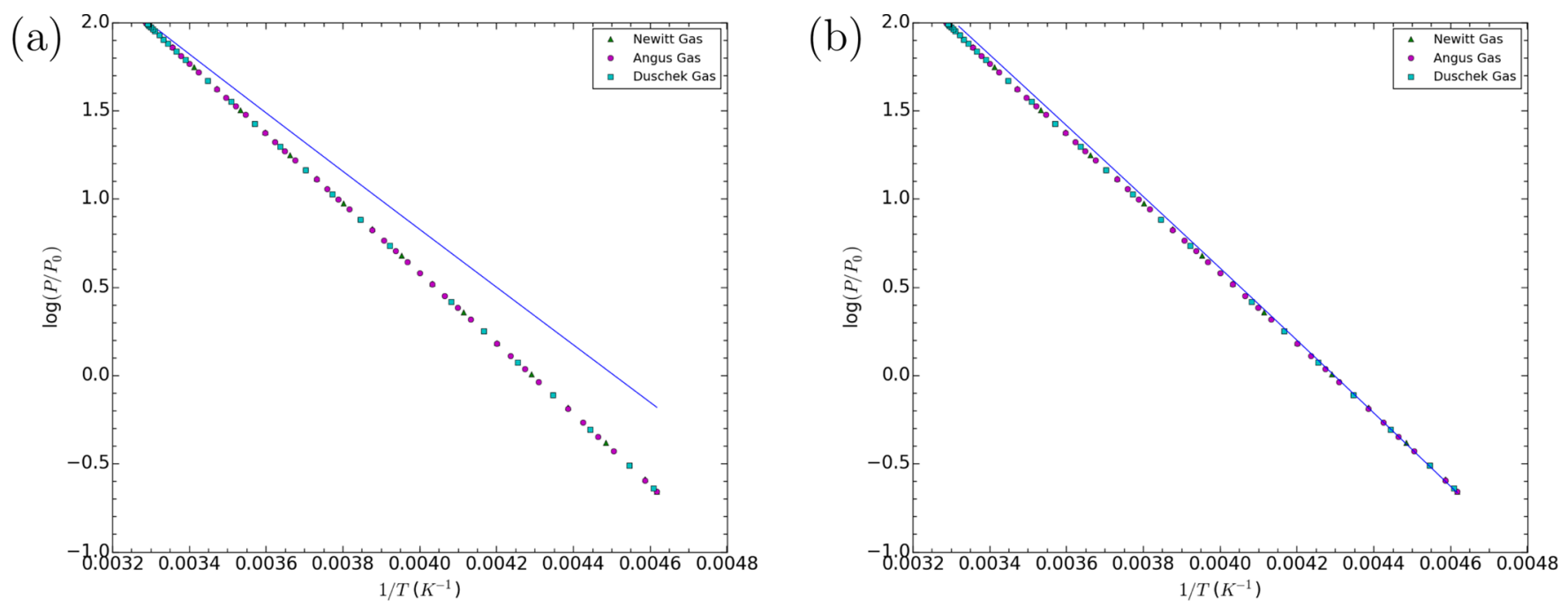

Figure 10. Logarithm of vapor pressure as a function of inverse temperature using (a) the characteristic parameters presented in this work and (b) the characteristic parameters of Kilpatrick and Chang. ${ }^{39}$ Symbols are experimental data from the sources indicated in the legends, and the solid lines are fits using the SL-EOS.

Comparing Table 1 with Figure 6 shows a correlation between the deviation from the experimental data and the scope of the ranges of pressure and temperature: the larger the temperature and pressure ranges considered, the better the fit. Figure 6, however, shows one seeming anomaly in that the Kilpatrick and Chang parameters have a relatively large temperature range and yet do not fit the experimental data well. This could possibly be explained by their proximity to the critical point. As mentioned, since SL theory is a mean-field theory, one does not expect predictions of thermodynamic data to be accurate near the critical point. ${ }^{20}$ Thus, fitting done in this region may decrease the accuracy of the results.

The SL-EOS for $\mathrm{CO}_{2}$ can also be analyzed with respect to other thermodynamic predictions, in keeping with IUPAC recommendations for EOS studies. ${ }^{72}$ In brief, the SL-EOS should be expected to give good results for quantities directly related to the PVT data to which it was fitted but not to quantities that depend on the internal degrees of freedom of the $\mathrm{CO}_{2}$ molecules. As originally pointed out by Sanchez and Lacombe, ${ }^{20}$ this is the case because internal degrees of freedom are not microscopically included in the model but rather are phenomenologically represented through the characteristic parameters; only translational degrees of freedom are explicitly incorporated. Thus, quantities like the thermal expansivity $\alpha$ and isothermal compressibility $\beta$ (see eqs $25 \mathrm{~b}$ and $26 \mathrm{~b}$ in ref 20) are predicted well using our recommended characteristic parameters values of $P^{*}=419.9 \mathrm{MPa}, T^{*}=341.8 \mathrm{~K}$, and $\rho^{*}=$ $1.397 \mathrm{~g} / \mathrm{cm}^{3}$, as shown in Figures $7 \mathrm{a}$ and $8 \mathrm{a}$. Other choices for the characteristic parameters fit the data less well. For example, the Kilpatrick and Chang predictions are shown in Figures $7 \mathrm{~b}$ and $8 \mathrm{~b}$. We note, however, a small overshoot and bump in the thermal expansivity and isothermal compressibility, respectively, in our fit at around $16 \mathrm{MPa}$ and $380 \mathrm{~K}$. The cause of this small deviation from the experimentally derived data is not clear. 

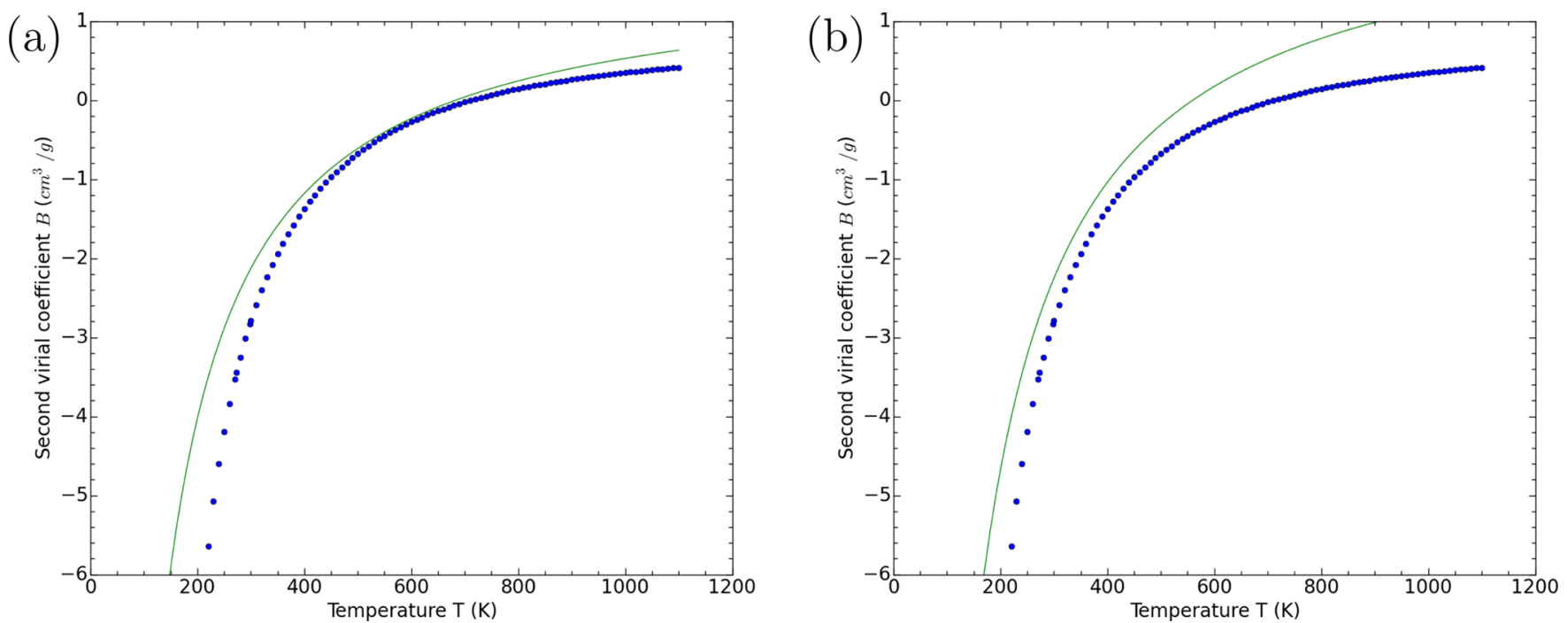

Figure 11. Second virial coefficient as a function of temperature using (a) the characteristic parameters presented in this work and (b) the characteristic parameters of Kilpatrick and Chang. ${ }^{39}$ Symbols are experimental results compiled by Angus et al.. ${ }^{64}$ and the solid lines are fits using the SL expression for $B$.

On the other hand, quantities directly related to the internal degrees of freedom of the $\mathrm{CO}_{2}$ molecules, such as the specific heats $c_{V}$ and $c_{P}$ and the Joule-Thomson inversion curve, which is related to $c_{P}$, should not be expected to correlate well. ${ }^{20}$ For all of the sets of characteristic parameters, including the set presented in this work, these quantities are not predicted correctly, not even qualitatively. For other quantities, such as the enthalpy of vaporization $\Delta H_{\text {vap }}$ and the vapor pressure, data are available mostly near the critical point, so the SL-EOS is not expected to perform well. Figures 9 and 10 show predictions of enthalpies of vaporization and vapor pressures, respectively, using the characteristic parameters of this work and those of Kilpatrick and Chang. In these cases, the Kilpatrick and Chang results follow the experimental data better than the predictions of this work. This is not surprising, since the Kilpatrick and Chang parameters were regressed to match vapor pressures. Since the mean-field SL theory should not correlate well with experiment for these data, this good agreement near the critical point for a mean-field theory is indicative of a problem. Specifically, achieving good agreement near the critical point means that the parameters are unable to predict correctly quantities away from the critical point and that the theory is being used as an interpolation formula in the region of the critical point rather than a predictive microscopic model.

The second virial coefficient $B$ of the SL-EOS (see eq $24 \mathrm{~d}$ of ref 20) adequately agrees with the experimental data compiled by Angus et al., ${ }^{64}$ as shown in Figure 11. For the characteristic parameters from this work, larger deviations are seen at lower temperatures surrounding the critical temperature, as expected. Again, the predictions using the parameters from this work are generally better than those of Kilpatrick and Chang. For all of the quantities examined in Figures 7-11, the predictions of Kilpatrick and Chang were chosen as points of comparison, but data from other groups behave similarly. Of course, the results of Xiong and Kiran and Nalawade et al. are competitive with the results of this work because of the similarity of the characteristic parameters.

Finally, it is relevant to question how the SL-EOS performs for $\mathrm{CO}_{2}$ compared with other equations of state. There are huge numbers of possible comparisons, but one obvious competitor is the Simha-Somcynsky EOS (SS-EOS). ${ }^{35,73}$ The SS-EOS is a cell-based model in which the cells are organized into a close-packed lattice. Like the SL-EOS, the SS-EOS is a hole-type theory in that each cell is either occupied by a single segment or is vacant. Unlike the SL-EOS, the segment does not occupy the entire cell, meaning that the free volume of the model is split into contributions from vacant cells and from occupied cells. The relative contributions to the free volume are determined by comparison with experiment, with multiple rules existing to accomplish this. The SS-EOS is a mean-field theory, so it will suffer the same limitations as the SL-EOS in the region of the critical point. The pure-component characteristic parameters are similar to those for the SL-EOS, but different interaction types are allowed, including square well ${ }^{35}$ and Lennard-Jones ${ }^{73}$ interactions. The underlying model of the SSEOS is somewhat more complicated than that underlying the SL-EOS, and the resulting formalism is significantly more complicated. The mixture formulation has been shown to be more successful than the SL-EOS one in some contexts, including polymeric foams. ${ }^{14,23-25}$ However, the pure-component parameters of the SL-EOS used for these comparisons may not be optimal, so this could contribute, at least in part, to the shortcomings of the mixture SL-EOS. ${ }^{26}$

\section{CONCLUSIONS}

It is found that the Sanchez-Lacombe pure-fluid characteristic parameters for carbon dioxide $P^{*}=419.9 \mathrm{MPa}, T^{*}=341.8 \mathrm{~K}$, and $\rho^{*}=1.397 \mathrm{~g} / \mathrm{cm}^{3}$ provide a good fit to PVT data and related quantities over large temperature and pressure ranges using a hole volume of $v^{*}=1.12 \times 10^{-23} \mathrm{~cm}^{3}$. As expected, properties that depend on the internal degrees of freedom of molecules, such as the specific heats, do not correlate well, because the SL-EOS does not include these degrees of freedom in the model. Properties directly related to the PVT data, however, such as the thermal expansivity and the isothermal compressibility, do correlate well with experiment. By analysis of the existing thermodynamic data for carbon dioxide over the temperature range of $216.58-1800 \mathrm{~K}$ and the pressure range of 0.5-66.57 $\mathrm{MPa}$, it is found that fitting practices play a nonnegligible role in the previous poor agreement between $P V T$ 
theory and experiment for the Sanchez-Lacombe equation of state for carbon dioxide. By fitting over the largest possible set of experimental data to date, it is found that a good fit can be achieved over much larger pressure and temperature ranges than previously thought possible. This should be true for many other pure substances, not just carbon dioxide.

\section{AUTHOR INFORMATION}

\section{Corresponding Author}

*E-mail: thompson@uwaterloo.ca.

\section{ORCID}

Russell B. Thompson: 0000-0002-6571-558X

\section{Funding}

This research was financially supported by the Natural Sciences and Engineering Research Council of Canada (NSERC) as well as by the Consortium for Cellular and Microcellular Plastics (CCMCP).

\section{Notes}

The authors declare no competing financial interest.

\section{REFERENCES}

(1) Span, R.; Wagner, W. A New Equation of State for Carbon Dioxide Covering the Fluid Region from the Triple-Point Temperature to $1100 \mathrm{~K}$ at Pressures up to $800 \mathrm{MPa}$. J. Phys. Chem. Ref. Data 1996, 25, 1509-1596.

(2) Cooper, A. I. Polymer Synthesis and Processing Using Supercritical Carbon Dioxide. J. Mater. Chem. 2000, 10, 207-234.

(3) Xiong, Y.; Kiran, E. Miscibility, Density and Viscosity of Poly(dimethylsiloxane) in Supercritical Carbon Dioxide. Polymer 1995, 36, 4817-4826.

(4) Tomasko, D. L.; Li, H.; Liu, D.; Han, X.; Wingert, M. J.; Lee, L. J.; Koelling, K. W. A Review of $\mathrm{CO}_{2}$ Applications in the Processing of Polymers. Ind. Eng. Chem. Res. 2003, 42, 6431-6456.

(5) Costeux, S. $\mathrm{CO}_{2}$-Blown Nanocellular Foams. J. Appl. Polym. Sci. 2014, 131, 41293.

(6) Boyère, C.; Jérôme, C.; Debuigne, A. Input of Supercritical Carbon Dioxide to Polymer Synthesis: An Overview. Eur. Polym. J. 2014, 61, 45-63.

(7) Sarikhani, K.; Jeddi, K.; Thompson, R. B.; Park, C. B.; Chen, P. Adsorption of Surface-Modified Silica Nanoparticles to the Interface of Melt Poly(lactic acid) and Supercritical Carbon Dioxide. Langmuir 2015, 31, 5571-5579.

(8) Sarikhani, K.; Jeddi, K.; Thompson, R. B.; Park, C. B.; Chen, P. Effect of Pressure and Temperature on Interfacial Tension of Poly Lactic Acid Melt in Supercritical Carbon Dioxide. Thermochim. Acta 2015, 609, 1-6.

(9) Xu, X.; Cristancho, D. E.; Costeux, S.; Wang, Z.-G. DensityFunctional Theory for Polymer-Carbon Dioxide Mixtures: A Perturbed-Chain SAFT Approach. J. Chem. Phys. 2012, 137, 054902.

(10) Li, G.; Wang, J.; Park, C. B.; Moulinie, P.; Simha, R. Comparison of SS-Based and SL-Based Estimation of Gas Solubility. In ANTEC 2004 Conference Proceedings; Society of Plastics Engineers: Brookfield Center, CT, 2004; pp 2566-2575.

(11) Mahmood, S. H.; Keshtkar, M.; Park, C. B. Determination of Carbon Dioxide Solubility in Polylactide Acid with Accurate PVT Properties. J. Chem. Thermodyn. 2014, 70, 13-23.

(12) Li, Z.-W.; Lu, Z.-Y.; Sun, Z.-Y.; Li, Z.-S.; An, L.-J. Calculating the Equation of State Parameters and Predicting the Spinodal Curve of Isotactic Polypropylene/Poly(ethylene-co-octene) Blend by Molecular Dynamics Simulations Combined with Sanchez-Lacombe Lattice Fluid Theory. J. Phys. Chem. B 2007, 111, 5934-5940.

(13) Li, G.; Gunkel, F.; Wang, J.; Park, C. B.; Altstädt, V. Solubility Measurements of $\mathrm{N}_{2}$ and $\mathrm{CO}_{2}$ in Polypropylene and Ethene/Octene Copolymer. J. Appl. Polym. Sci. 2007, 103, 2945-2953.

(14) Hasan, M. M.; Li, Y. G.; Li, G.; Park, C. B.; Chen, P. Determination of Solubilities of $\mathrm{CO}_{2}$ in Linear and Branched
Polypropylene Using a Magnetic Suspension Balance and a PVT Apparatus. J. Chem. Eng. Data 2010, 55, 4885-4895.

(15) Li, G.; Li, H.; Wang, J.; Park, C. B. Investigating the Solubility of $\mathrm{CO}_{2}$ in Polypropylene Using Various EOS Models. Cell. Polym. 2006, $25,237-248$

(16) Liao, X.; Li, Y. G.; Park, C. B.; Chen, P. Interfacial Tension of Linear and Branched PP in Supercritical Carbon Dioxide. J. Supercrit. Fluids 2010, 55, 386-394.

(17) Park, H.; Park, C. B.; Tzoganakis, C.; Tan, K. H.; Chen, P. Surface Tension Measurement of Polystyrene Melts in Supercritical Carbon Dioxide. Ind. Eng. Chem. Res. 2006, 45, 1650-1658.

(18) Walker, T. A.; Colina, C. M.; Gubbins, K. E.; Spontak, R. J. Thermodynamics of Poly(dimethylsiloxane)/Poly(ethylmethylsiloxane) (PDMS/PEMS) Blends in the Presence of High-Pressure $\mathrm{CO}_{2}$. Macromolecules 2004, 37, 2588-2595.

(19) Sanchez, I. C.; Lacombe, R. H. Theory of Liquid-Liquid and Liquid-Vapour Equilibria. Nature 1974, 252, 381-383.

(20) Sanchez, I. C.; Lacombe, R. H. An Elementary Molecular Theory of Classical Fluids. Pure fluids. J. Phys. Chem. 1976, 80, 23522362.

(21) Lacombe, R. H.; Sanchez, I. C. Statistical Thermodynamics of Fluid Mixtures. J. Phys. Chem. 1976, 80, 2568-2580.

(22) Li, G.; Wang, J.; Park, C. B.; Simha, R. Measurement of Gas Solubility in Linear/Branched PP Melts. J. Polym. Sci., Part B: Polym. Phys. 2007, 45, 2497-2508.

(23) Li, Y. G.; Park, C. B.; Li, H. B.; Wang, J. Measurement of the PVT Property of $\mathrm{PP} / \mathrm{CO}_{2}$ Solution. Fluid Phase Equilib. 2008, 270, $15-22$.

(24) Mahmood, S. H.; Xin, C. L.; Lee, J. H.; Park, C. B. Study of Volume Swelling and Interfacial Tension of the Polystyrene-Carbon Dioxide-Dimethyl Ether System. J. Colloid Interface Sci. 2015, 456, 174-181.

(25) Li, Y. G.; Park, C. B. Effects of Branching on the PressureVolume-Temperature Behaviors of $\mathrm{PP} / \mathrm{CO}_{2}$ Solutions. Ind. Eng. Chem. Res. 2009, 48, 6633-6640.

(26) Bashir, M. A.; Al-haj Ali, M.; Kanellopoulos, V.; Seppälä, J.; Kokko, E.; Vijay, S. The Effect of Pure Component Characteristic Parameters on Sanchez-Lacombe Equation-of-State Predictive Capabilities. Macromol. React. Eng. 2013, 7, 193-204.

(27) Kim, J.-H.; Paxton, T. E.; Tomasko, D. L. Microencapsulation of Naproxen Using Rapid Expansion of Supercritical Solutions. Biotechnol. Prog. 1996, 12, 650-661.

(28) Kim, Y.; Park, C. B.; Chen, P.; Thompson, R. B. Origins of the Failure of Classical Nucleation Theory for Nanocellular Polymer Foams. Soft Matter 2011, 7, 7351-7358.

(29) Kim, Y.; Park, C. B.; Chen, P.; Thompson, R. B. Towards Maximal Cell Density Predictions for Polymeric Foams. Polymer 2011, 52, 5622-5629.

(30) Kim, Y.; Park, C. B.; Chen, P.; Thompson, R. B. Maximal Cell Density Predictions for Compressible Polymer Foams. Polymer 2013, 54, 841-845.

(31) Park, H.; Thompson, R. B.; Lanson, N.; Tzoganakis, C.; Park, C. B.; Chen, P. Effect of Temperature and Pressure on Surface Tension of Polystyrene in Supercritical Carbon Dioxide. J. Phys. Chem. B 2007, $111,3859-3868$.

(32) Thompson, R. B.; MacDonald, J. R.; Chen, P. Origin of Change in Molecular-Weight Dependence for Polymer Surface Tension. Phys. Rev. E 2008, 78, 030801.

(33) Hong, K. M.; Noolandi, J. Conformational Entropy Effects in a Compressible Lattice Fluid Theory of Polymers. Macromolecules 1981, 14, 1229-1234.

(34) Thompson, R. B.; Park, C. B.; Chen, P. Reduction of Polymer Surface Tension by Crystallized Polymer Nanoparticles. J. Chem. Phys. 2010, 133, 144913.

(35) Simha, R.; Somcynsky, T. On the Statistical Thermodynamics of Spherical and Chain Molecule Fluids. Macromolecules 1969, 2, 342350. 
(36) Hariharan, R.; Freeman, B. D.; Carbonell, R. G.; Sarti, G. C. Equation of State Predictions of Sorption Isotherms in Polymeric Materials. J. Appl. Polym. Sci. 1993, 50, 1781-1795.

(37) Cao, G.-P.; Liu, T.; Roberts, G. W. Predicting the Effect of Dissolved Carbon Dioxide on the Glass Transition Temperature of Poly(acrylic acid). J. Appl. Polym. Sci. 2010, 115, 2136-2143.

(38) Garg, A.; Gulari, E.; Manke, C. W. Thermodynamics of Polymer Melts Swollen with Supercritical Gases. Macromolecules 1994, 27, $5643-5653$.

(39) Kilpatrick, P. K.; Chang, S.-H. Saturated Phase Equilibria and Parameter Estimation of Pure Fluids with Two Lattice-Gas Models. Fluid Phase Equilib. 1986, 30, 49-56.

(40) Kiszka, M. B.; Meilchen, M. A.; McHugh, M. A. Modeling HighPressure Gas-Polymer Mixtures Using the Sanchez-Lacombe Equation of State. J. Appl. Polym. Sci. 1988, 36, 583-597.

(41) Nalawade, S. P.; Picchioni, F.; Janssen, L. P. B. M.; Patil, V. E.; Keurentjes, J. T. F.; Staudt, R. Solubilities of Sub- and Supercritical Carbon Dioxide in Polyester Resins. Polym. Eng. Sci. 2006, 46, 643649.

(42) Pope, D. S.; Sanchez, I. C.; Koros, W. J.; Fleming, G. K. Statistical Thermodynamic Interpretation of Sorption/Dilation Behavior of Gases in Silicone Rubber. Macromolecules 1991, 24, 1779-1783.

(43) Wang, N.-H.; Hattori, K.; Takishima, S.; Masuoka, H. Measurement and Prediction of Vapor-Liquid Equilibrium Ratios for Solutes at Infinite Dilution in $\mathrm{CO}_{2}+$ Polyvinyl Acetate System at High Pressures. Kagaku Kogaku Ronbunshu 1991, 17, 1138-1145.

(44) Rodgers, P. A. Pressure-Volume-Temperature Relationships for Polymeric Liquids: A Review of Equations of State and Their Characteristic Parameters for 56 Polymers. J. Appl. Polym. Sci. 1993, 48, 1061-1080.

(45) Pathria, R. K. Statistical Mechanics, 2nd ed.; Butterworth Heinemann: Boston, 1996; pp 356-359.

(46) Binney, J. J.; Dowrick, N. J.; Fisher, A. J.; Newman, M. E. J. The Theory of Critical Phenomena: An Introduction to the Renormalization Group, 1st ed.; Oxford University Press: New York, 1992; pp 176177.

(47) Gauter, K.; Heidemann, A. A Proposal for Parametrizing the Sanchez-Lacombe Equation of State. Ind. Eng. Chem. Res. 2000, 39, $1115-1117$.

(48) Krenz, R. A.; Laursen, T.; Heidemann, R. A. The Modified Sanchez-Lacombe Equation of State Applied to Polydisperse Polyethylene Solutions. Ind. Eng. Chem. Res. 2009, 48, 10664-10681.

(49) Péneloux, A.; Rauzy, E.; Fréze, R. A. A Consistent Correction for Redlich-Kwong-Soave Volumes. Fluid Phase Equilib. 1982, 8, 7-23.

(50) Machida, H.; Sato, Y.; Smith, R. L. Simple Modification of the Temperature Dependence of the Sanchez-Lacombe Equation of State. Fluid Phase Equilib. 2010, 297, 205-209.

(51) Iguchi, M.; Machida, H.; Sato, Y.; Smith, R. L. Correlation of Supercritical $\mathrm{CO}_{2}$-Ionic Liquid Vapor-Liquid Equilibria with the $\epsilon^{*}$ Modified Sanchez-Lacombe Equation of State. Asia-Pac. J. Chem. Eng. 2012, 7, S95-S100.

(52) Ahn, S. J. In Lecture Notes in Computer Science, Vol. 1070; Springer: Berlin, 2004.

(53) Marquardt, D. W. An Algorithm for Least-Squares Estimation of Nonlinear Parameters. J. Soc. Ind. Appl. Math. 1963, 11, 431-441.

(54) Zoller, P. Analysis of the Equation of State of Polymer Melts in Terms of the Ising Fluid Model. J. Polym. Sci., Polym. Phys. Ed. 1980, 18, 157-160.

(55) Pottiger, M. T.; Laurence, R. L. The P-V-T Behavior of Polymeric Liquids Represented by the Sanchez-Lacombe Equation of State. J. Polym. Sci., Polym. Phys. Ed. 1984, 22, 903-907.

(56) Doghieri, F.; Sarti, G. C. Nonequilibrium Lattice Fluids: A Predictive Model for the Solubility in Glassy Polymers. Macromolecules 1996, 29, 7885-7896.

(57) Funami, E.; Taki, K.; Ohshima, M. Density Measurement of Polymer $/ \mathrm{CO}_{2}$ Single-Phase Solution at High Temperature and Pressure Using a Gravimetric Method. J. Appl. Polym. Sci. 2007, 105, 3060-3068.
(58) Arce, P. F.; Aznar, M. Modeling of Thermodynamic Behavior of PVT Properties and Cloud Point Temperatures of Polymer Blends and Polymer Blend+Carbon Dioxide Systems Using Non-Cubic Equations of State. Fluid Phase Equilib. 2009, 286, 17-27.

(59) Sen Roy, S.; Guria, S. Estimation of Regression Parameters in the Presence of Outliers in the Response. Statistics 2009, 43, 531-539.

(60) Vogt, F. A Self-Guided Search for Good Local Minima of the Sum-of-Squared-Error in Nonlinear Least Squares Regression. J. Chemom. 2015, 29, 71-79.

(61) Vidaurre, G.; Vasquez, V. R.; Whiting, W. B. Robustness of Nonlinear Regression Methods under Uncertainty: Applications in Chemical Kinetics Models. Ind. Eng. Chem. Res. 2004, 43, 1395-1404.

(62) Bonilla-Petriciolet, A. On The Capabilities and Limitations of Harmony Search for Parameter Estimation in Vapor-Liquid Equilibrium Modeling. Fluid Phase Equilib. 2012, 332, 7-20.

(63) Bondi, A. Physical Properties of Molecular Crystals, Liquids, and Glasses; Wiley: New York, 1968; p 502.

(64) Angus, S.; Armstrong, B.; de Rueck, K. M. International Thermodynamic Tables of the Fluid State 3: Carbon Dioxide; Pergamon Press: Oxford, U.K., 1976.

(65) Vukalovich, M. P.; Altunin, V. V. Thermophysical Properties of Carbon Dioxide; Collets: Wellingborough, U.K., 1968.

(66) Canjar, L. N.; Manning, F. S. Thermodynamic Properties and Reduced Correlations for Gases; Gulf Publishing Company: Houston, TX, 1967; p 212.

(67) Michels, A.; Blaisse, B.; Michels, C. The Isotherms of $\mathrm{CO}_{2}$ in the Neighbourhood of the Critical Point and Round the Coexistence Line. Proc. R. Soc. London, Ser. A 1937, 160, 358-375.

(68) Vargaftik, N. B. Tables on the Thermophysical Properties of Liquids and Gases: in Normal and Dissociated States, 2nd ed.; Hemisphere Publishing Corp.: Washington, DC, 1975; p 758.

(69) NIST. Thermophysical Properties of Fluid Systems. http:// webbook.nist.gov/chemistry/fluid/ (accessed Jan 1, 2016).

(70) Duschek, W.; Kleinrahm, R.; Wagner, W. Measurement and Correlation of the (Pressure, Density, Temperature) Relation of Carbon Dioxide I. The Homogeneous Gas and Liquid Regions in the Temperature Range from 217 to $340 \mathrm{~K}$ at Pressures up to $9 \mathrm{MPa}$. J. Chem. Thermodyn. 1990, 22, 827-840.

(71) Duschek, W.; Kleinrahm, R.; Wagner, W. Measurement and Correlation of the (Pressure, Density, Temperature) Relation of Carbon Dioxide II. Saturated-Liquid and Saturated-Vapour Densities and the Vapour Pressure Along the Entire Coexistence Curve. J. Chem. Thermodyn. 1990, 22, 841-864.

(72) Deiters, U. K.; De Reuck, K. M. Guidelines for Publication of Equations of State - I. Pure fluids. Fluid Phase Equilib. 1999, 161, 205219.

(73) Xie, H.; Nies, E.; Stroeks, A.; Simha, R. Some Considerations on Equation of State and Phase-Relations - Polymer-Solutions and Blends. Polym. Eng. Sci. 1992, 32, 1654-1664. 